\title{
Avaliação dos resultados da atenção multiprofissional sobre o controle glicêmico, perfil lipídico e estado nutricional de diabéticos atendidos em nível primário
}

\section{Evaluating the results of multiprofessional health care \\ on the glucemic control, lipid profile, and nutritional status \\ of diabetic patients assisted at primary level}

Maria da Conceição Rosado BATISTA ${ }^{1}$

Silvia Eloiza PRIORE ${ }^{1}$

Lina Enriqueta Frandsen Paez de Lima ROSADO ${ }^{1}$

Adelson Luiz Araújo TINÔCO ${ }^{1}$

Sylvia do Carmo Castro FRANCESCHINI

\section{R E S U M O}

\section{Objetivo}

Avaliar o resultado da intervenção multiprofissional sobre o controle glicêmico, o perfil lipídico e o estado nutricional de diabéticos atendidos em nível primário em Belo Horizonte, MG.

\section{Métodos}

Analisaram-se a glicemia, o índice de massa corporal e o perfil lipídico de 190 diabéticos tipo 2 (31 a 83 anos), atendidos no período de 1993 a 2000. Os dados foram coletados no momento inicial (primeira consulta de nutrição) e após três meses, tendo alguns sido coletados também uma terceira vez (6 meses após a primeira consulta).

\section{Resultados}

Inicialmente foram observados valores médios e medianos elevados, para índice de massa corporal, glicemia de jejum, colesterol total, LDL-c e triglicerídeos. Nos primeiros três meses após a intervenção, houve redução do índice de massa corporal (de 28,3 para 27,8kg/m²), glicemia de jejum (de 178,5 para 135,0mg/dL), colesterol total (de 251,0 para 230,0mg/dL), LDL-c (de 185,5 para 159mg/dL) e triglicerídeos (de 243,0 para 190,0mg/dL), não ocorrendo alteração dos níveis de HDL-c. Os pacientes com índice de massa corporal $<30 \mathrm{~kg} / \mathrm{m}^{2}$ e os com diabetes de manifestação mais recente, obtiveram melhores resultados.

\footnotetext{
1 Departamento de Nutrição e Saúde, Universidade Federal de Viçosa. Campus Universitário, 36570-000, Viçosa, MG, Brasil. Correspondência para/Correspondence to: M.C.R. BATISTA. E-mail: <mcrbatista@bol.com.br>.
} 


\section{Conclusão}

Os resultados deste estudo indicam que o atendimento multiprofissional é importante para o controle do diabetes, devendo ser iniciado precocemente.

Termos de indexação: glicemia, diabetes mellitus, estado nutricional, intervenção nutricional.

\section{A B S T R A C T}

\section{Objective}

This work evaluates the multiprofessional intervention results on glucemic control, lipid profile, and nutritional status of diabetic patients, who received primary level health care in Belo Horizonte, state of Minas Gerais, Brazil.

\section{Methods}

An examination was carried out, to evaluate the levels of glucemia, body mass index, and lipid profile of 190 type-2 diabetic patients (31 to 83 years old), assisted during the period of 1993 to the year 2000. The data were collected at the ${ }^{1 \text { st. }}$. consultation on nutrition, and after three months, whereas some data were also collected at a $3^{r d}$. moment (6 months after the initial consultation).

\section{Results}

Initially, the registered data were the high average and medium values for body mass index, fasting glucemia, total cholesterol, LDL-c and triglycerides. Over the first three months after intervention, a significant reduction occurred in the body mass index (from 28.3 to $27.8 \mathrm{~kg} / \mathrm{m}^{2}$ ), fasting glucemia levels (from 178.5 to $135.0 \mathrm{mg} / \mathrm{dL}$ ), total cholesterol (from 251.0 to $230.0 \mathrm{mg} / \mathrm{dL}$ ), LDL-c (from 185.5 to $159 \mathrm{mg} / \mathrm{dL}$ ) and triglycerides (from 243.0 to $190.0 \mathrm{mg} / \mathrm{dL}$ ); however, no alteration occurred in the HDL-c levels. The patients with body mass index $<30 \mathrm{~kg} / \mathrm{m}^{2}$, as well as those with a more recent history of diabetes obtained better outcomes.

\section{Conclusion}

This study's results allow us to conclude that multiprofessional health care is fundamental in controlling diabetes, and such care should begin as soon as possible.

Indexing terms: blood glucose, diabetes mellitus, nutritional status, nutritional intervention.

\section{N T R O D U Ç Ã O}

O diabetes mellitus tipo 2 é uma síndrome heterogênea que resulta de defeitos na secreção e na ação da insulina', sendo que a patogênese de ambos os mecanismos está relacionada a fatores genéticos e ambientais². Sua incidência e prevalência vêm aumentando em várias populações, tornando-se uma das doenças mais prevalentes no mundo ${ }^{3}$.

No Brasil, dados do Estudo Multicêntrico de Diabetes, realizado no período de 1986 a 1988, mostraram que o diabetes acomete cerca de 7,6\% da população entre 30 e 69 anos de idade. Mais de $50,0 \%$ dos diagnosticados não sabiam ser portadores da doença, enquanto $24,0 \%$ dos reconhecidamente diabéticos não faziam qualquer tipo de tratamento ${ }^{4}$.
A Campanha Nacional de Detecção de Casos Suspeitos de Diabetes, realizada em março e abril de 2001, identificou 699265 casos suspeitos, que correspondem a 15\% da população testada ${ }^{5}$.

O diabetes está associado ao aumento da mortalidade e ao alto risco de desenvolvimento de complicações micro e macro-vasculares, como também de neuropatias. Pode resultar em cegueira, insuficiência renal e amputações de membros, sendo responsável por gastos excessivos em saúde e substancial redução da capacidade de trabalho e da expectativa de vida ${ }^{6}$. No entanto, ensaios clínicos randomizados têm demonstrado que, pacientes mantidos em controle clínico e metabólico, apresentam retardo no aparecimento e/ou progressão de complicações crônicas . 
O manejo do paciente diabético deve ser feito dentro de um sistema hierarquizado de saúde, sendo sua base o nível primário ${ }^{8}$. Porém, em nosso meio, pouco se conhece a respeito do processo de cuidado neste nível de assistência à saúde ${ }^{3}$.

Com o intuito de contribuir para esse conhecimento, o presente estudo avalia os resultados obtidos com a atenção clínico-nutricional, sobre o controle glicêmico, o perfil lipídico e o estado nutricional de diabéticos tipo 2 , atendidos em unidades básicas de saúde da periferia de Belo Horizonte.

\section{MÉTO D O S}

Participaram deste estudo 190 pacientes com diabetes tipo 2, atendidos em duas unidades básicas de saúde da periferia de Belo Horizonte, no período de 1993 a 2000.

Nestas unidades, as atividades de assistência ao diabético consistiam em consultas individuais com clínico, nutricionista e, quando necessário, com enfermeiro, assistente social, psicólogo e/ou dentista. Estes profissionais ofereciam também orientações, em grupos operativos de educação em diabetes, aos quais os pacientes possuíam livre acesso. Sempre que necessário, os pacientes eram referenciados à atenção secundária de outros profissionais: endocrinologista, oftamologista (anualmente), nefrologista, angiologista e outros.

No primeiro atendimento nutricional, era realizada uma anamnese alimentar detalhada e, baseando-se nela, procedia-se à orientação dietética. No segundo atendimento, o paciente recebia uma prescrição dietética individualizada, de acordo com sua idade, sexo, peso e atividade física.

Os critérios de inclusão para o estudo foram: idade superior a 30 anos e ter tido pelo menos duas consultas com o nutricionista. Foram excluídos os pacientes que se tornaram diabéticos após os 30 anos, mas cuja doença apresentava características de diabetes tipo 1, tais como o início abrupto e, desde o início, a necessidade de insulina.

Os dados foram coletados das fichas de atendimento nutricional e organizados em dois momentos diferentes: primeiro atendimento (primeira consulta) e segundo atendimento (mediana de três meses após a primeira consulta). Algumas variáveis foram avaliadas também em um terceiro momento (mediana de seis meses após a primeira consulta).

Devido à coleta de dados ter sido realizada a partir de fichas de acompanhamento pertencentes a um serviço de saúde que não visava análise científica, o tamanho da amostra mostrou-se diferente para cada variável, pois os pacientes não possuíam todos os dados em todas as consultas, fato que dependeu da rotina e da realidade do serviço.

Os pacientes foram pesados e medidos pelo mesmo profissional, em balança com antropômetro acoplado, tipo plataforma, com capacidade de $150 \mathrm{~kg}$ e precisão de $100 \mathrm{~g}$.

Os exames bioquímicos foram realizados por laboratórios municipais e conveniados. As coletas de sangue foram feitas após jejum, de no mínimo 8 horas para verificar glicemia de jejum, e de 12 horas para lípides séricos.

O estado nutricional foi avaliado por meio do índice de massa corporal (IMC), e os indivíduos classificados como baixo peso (IMC $<18,5 \mathrm{~kg} / \mathrm{m}^{2}$ ), peso normal $\left(18,5-24,9 \mathrm{~kg} / \mathrm{m}^{2}\right)$, sobrepeso ou pré-obeso $\left(25-29,9 \mathrm{~kg} / \mathrm{m}^{2}\right)$, além de níveis de obesidade: classe $1\left(30-34,9 \mathrm{~kg} / \mathrm{m}^{2}\right)$, classe 2 $\left(35-39,9 \mathrm{~kg} / \mathrm{m}^{2}\right)$ e classe $3\left(\geq 40 \mathrm{~kg} / \mathrm{m}^{2}\right)^{9}$.

Utilizaram-se como referência para controle glicêmico e perfil lipídico os valores preconizados pelo Consenso Brasileiro de Diabetes, da Sociedade Brasileira de Diabetes (2000) ${ }^{10}$. A glicemia de jejum foi considerada normal até $110 \mathrm{mg} / \mathrm{dL}$ e aceitável até $126 \mathrm{mg} / \mathrm{dL}$. Para os lípides séricos, consideraram-se ideais os seguintes níveis: de colesterol total, inferior a $200 \mathrm{mg} / \mathrm{dL}$; de $\mathrm{HDL}$, superior a $45 \mathrm{mg} / \mathrm{dL}$; de $\mathrm{LDL}$, inferior a $100 \mathrm{mg} / \mathrm{dL}$; de triglicérides, inferior a $150 \mathrm{mg} / \mathrm{dL}^{10}$. 
Não foi possível avaliar a glicemia pós-prandial e a hemoglobina glicada. A primeira, devido à pouca freqüência com que foram realizados estes exames (para apenas quatro indivíduos); a segunda, devido à grande variabilidade de metodologias para quantificar a hemoglobina glicada, sendo que, geralmente, os pacientes realizavam este exame em diferentes laboratórios, com valores de referência diversos.

Quanto à atividade física regular, foi considerado como tal, qualquer exercício realizado pelo menos três vezes por semana, com duração de no mínimo 30 minutos.

Os dados foram processados no programa Epi Info, versão 6,04 $4^{11}$. O programa Sigma Stat ${ }^{12}$ foi utilizado para comparar os valores medianos obtidos no primeiro e segundo momentos, tendo sido usado para tal o teste de Wilcoxon. Para comparar dois grupos independentes, foi utilizado o teste de Mann-Whitney e, para comparar a freqüência de glicemia aceitável antes e após intervenção, utilizou-se o teste do $\chi^{2}$. Adotou-se como nível de significância, o valor 0,05 ou 5\%.

\section{RESULTADOS E DISCUSSÃO}

A mediana de idade dos pacientes foi de 57 (31-83) anos, sendo a maioria do sexo feminino. Os indivíduos acima dos 50 anos somavam $73,7 \%$ do total, sendo prevalente a faixa etária de 60 a 69 anos (Tabela 1).

A idade mediana dos pacientes ao iniciarem o acompanhamento nutricional, foi semelhante à idade média encontrada em estudo transversal, realizado na Finlândia em 1999 $(58 \pm 17 \text { anos })^{13}$. Entretanto, essa idade mediana foi um pouco superior à de 54,6 \pm 10,1 anos, verificada no estudo de intervenção educativa, realizado em 10 países da América Latina por Gagliardino \& Etchegoyen ${ }^{14}$.

No presente estudo, a maior parte dos diabéticos encontrava-se na faixa etária de 50 a 69 anos (63,5\%), o que se aproxima do observado no estudo de Araújo et al. ${ }^{3},(65,7 \%)$, realizado em Pelotas, RS.

Em relação à freqüência de indivíduos na faixa etária igual ou superior a 70 anos, o presente

Tabela 1. Características demográficas e da doença dos pacientes diabéticos no início do acompanhamento nutricional. Belo Horizonte, 1993 a 2000.

\begin{tabular}{|c|c|c|}
\hline Características & $n$ & Percentual \\
\hline \multicolumn{3}{|l|}{ Idade (anos) } \\
\hline $30 \vdash 39$ & 11 & 5,8 \\
\hline $40 \vdash 49$ & 39 & 20,5 \\
\hline $50 \vdash 59$ & 57 & 30,0 \\
\hline $60 \vdash 69$ & 63 & 33,2 \\
\hline$\geq 70$ & 20 & 10,5 \\
\hline \multicolumn{3}{|l|}{ Sexo } \\
\hline Masculino & 41 & 21,6 \\
\hline Feminino & 149 & 78,4 \\
\hline \multicolumn{3}{|c|}{ Tempo de diagnóstico de DM (meses) } \\
\hline $1 \vdash 60$ & 117 & 72,6 \\
\hline $61 \vdash 120$ & 22 & 13,7 \\
\hline $121 \vdash 180$ & 10 & 6,2 \\
\hline$>180$ & 12 & 7,5 \\
\hline \multicolumn{3}{|l|}{ Tipo de medicamento } \\
\hline Nenhum & 84 & 48,0 \\
\hline Medicamento oral & 77 & 44,0 \\
\hline Insulina & 13 & 7,4 \\
\hline Insulina + medicamento oral & 1 & 0,6 \\
\hline \multicolumn{3}{|l|}{ Atividade física } \\
\hline $\operatorname{sim}$ & 20 & 14,7 \\
\hline Não & 116 & 85,3 \\
\hline
\end{tabular}


estudo mostra prevalência quatro vezes menor à encontrada por Schumacher et $\mathrm{al}^{15}$. em um estado norte-americano $(41,6 \%)$. Este fato, tanto pode sugerir que haja uma sobrevida maior no grupo populacional americano, quanto uma diferença na sua pirâmide etária geral.

Observou-se neste estudo, a prevalência de mulheres em acompanhamento nas Unidades de Saúde, em número quatro vezes maior que o de homens, enquanto que, no Estudo Multicêntrico (1986-1988), a freqüência foi semelhante entre homens e mulheres, respectivamente 7,5 e $7,6 \%{ }^{4}$. Araújo et al. ${ }^{3}$ e Assunção et al. ${ }^{16}$, em estudos com diabéticos atendidos em nível primário, em Pelotas, RS, também encontraram maior prevalência entre as mulheres, respectivamente $70,6 \%$ (2,4 vezes maior que os homens) e $76,1 \%$ (3,1 vezes maior).

Goldenberg et al. ${ }^{17}$, avaliando o diabetes auto-referido no município de São Paulo, encontraram entre os homens, a maior proporção de desconhecimento da presença da doença. Verificaram também que, entre os pacientes diagnosticados, as mulheres freqüentaram mais os serviços de saúde, no período compreendido pelos últimos doze meses.

A maior concentração de mulheres utilizando os serviços de atenção primária em diabetes, tanto pode sugerir uma maior preocupação destas com sua própria saúde, quanto uma maior facilidade de acesso aos serviços. Isto porque, desde a perspectiva da divisão sexual do trabalho, a tarefa de providenciar assistência médica à família é vista como atributo feminino, vindo a aumentar o contato da mulher com o serviço de saúde e a garantir acesso facilitado ${ }^{18}$. Este fato é favorável ao sexo feminino, uma vez que há evidências de que, o maior contato com os serviços médicos resulta em maior proteção à saúde ${ }^{19}$.

Observou-se que, ao iniciar o acompanhamento nutricional, a maior parte dos pacientes $(72,6 \%)$ relatava ser recente a manifestação da doença, com um tempo de duração igual ou inferior a cinco anos (Tabela1). Importa também salientar que $46 \%(n=74)$ dos pacientes tinham recebido diagnóstico de diabetes há apenas seis meses ou menos. Estes dados contribuem para enfatizar a importância de buscar melhores resultados das ações educativas sobre o diabetes, prevenindo ou postergando o aparecimento de complicações.

No entanto, é importante salientar que os outros $27,4 \%$ dos pacientes, apesar de já terem a doença diagnosticada há mais de cinco anos, nunca haviam passado anteriormente por uma consulta de nutrição, que é um aspecto importante para o controle do diabético.

Ao iniciar o acompanhamento, grande parte dos pacientes $(48,0 \%)$ não utilizava nenhuma medicação para diabetes e apenas $14,7 \%$ realizavam alguma atividade física regular (Tabela 1).

Ao iniciarem o acompanhamento nutricional, apenas $8,0 \%$ dos pacientes usavam insulina, resultado semelhante ao encontrado por Araújo et al. ${ }^{3}$, em cujo estudo verificou-se que apenas $10,4 \%$ dos pacientes a utilizavam. Já em estudo realizado na Finlândia ${ }^{13}$, 50,0\% eram dependentes de insulina; entretanto, este alto percentual, provavelmente, se deve ao maior número de pessoas diagnosticadas há mais de 10 anos (56,3\%), enquanto que, no presente estudo, os indivíduos com mais de 10 anos representam apenas $13,7 \%$.

Inicialmente, foram observados valores médios e medianos, elevados para IMC, glicemia de jejum, colesterol total, LDL-c e triglicerídeos (Tabela 2), não havendo diferença significante destes valores em relação ao sexo, nem à idade (adultos ou idosos), exceto para IMC, cujos índices foram mais elevados para as mulheres do que para os homens, $29,12 \pm 4,99 \mathrm{~kg} / \mathrm{m}^{2} \mathrm{e}$ $27,50 \pm 4,06 \mathrm{~kg} / \mathrm{m}^{2}(p<0,05)$.

Em relação ao estado nutricional, apenas $20,0 \%$ dos pacientes apresentaram peso normal à primeira consulta, enquanto $44,7 \%$ tinham sobrepeso e $34,8 \%$ eram obesos. No primeiro momento, a obesidade (IMC $\geq 30)$ relacionou-se apenas ao HDL-c baixo, sendo a mediana dos 
obesos de 39,0 (24-58) mg/dL e a dos não-obesos, de 50,0 (37-70) mg/dL $(p<0,05)$.

No presente estudo, a alta prevalência de obesidade vem confirmar a presença desta como um fator de risco para o diabetes. Os valores médios de IMC, mais elevados entre as mulheres, estão de acordo com os resultados de estudos de avaliação nutricional realizados em diversas regiões do Brasil, os quais demonstram elevada prevalência de obesidade entre as mulheres, principalmente entre aquelas de populações menos favorecidas ${ }^{20}$.

Neste estudo, apenas o HDL-c baixo esteve relacionado ao IMC maior ou igual a 30. Entre- tanto, outros estudos observaram que a obesidade está relacionada também a outras alterações, tais como os altos níveis de triglicerídeos e a maior prevalência de hipertensão, sendo esta relação mais evidente no sexo masculino ${ }^{21}$.

Quanto à glicemia de jejum, no primeiro atendimento não houve diferença significante entre obesos e não obesos; porém, nos momentos seguintes, após a intervenção dietética, a glicemia foi significantemente menor nos pacientes nãoobesos, tanto no segundo, quanto no terceiro atendimento (Figura 1).

Relacionando-se obesidade e níveis glicêmicos, no primeiro momento, quando os

Tabela 2. Estado nutricional, controle glicêmico e lipídeos séricos no momento inicial do acompanhamento nutricional. Belo Horizonte, 1993 a 2000.

\begin{tabular}{lcrrrrr}
\hline Variáveis & $\mathrm{n}$ & $\mathrm{M} \pm \mathrm{DP}$ & Mínimo & Mediana & Máximo \\
\hline IMC (kg/m²) & 190 & $28,8 \pm 4,8$ & 14,7 & 28,3 & 45,9 \\
Glicemia jejum (mg/dL) & 190 & $199,2 \pm 76,3$ & 82,0 & 178,5 & 470,0 \\
Colesterol total (mg/dL) & 91 & $228,0 \pm 46,6$ & 127,0 & 222,0 & 371,0 \\
HDL-c (mg/dL) & 20 & $46,2 \pm 13,0$ & 24,0 & 45,0 & 70,0 \\
LDL-c (mg/dL) & 27 & $154,3 \pm 42,3$ & 57,0 & 157,0 & 255,0 \\
Triglicerídeos (mg/dL) & 79 & $224,1 \pm 141,0$ & 70,0 & 181,0 & 788,0 \\
\hline
\end{tabular}

M= Média; DP = Desvio-Padrão

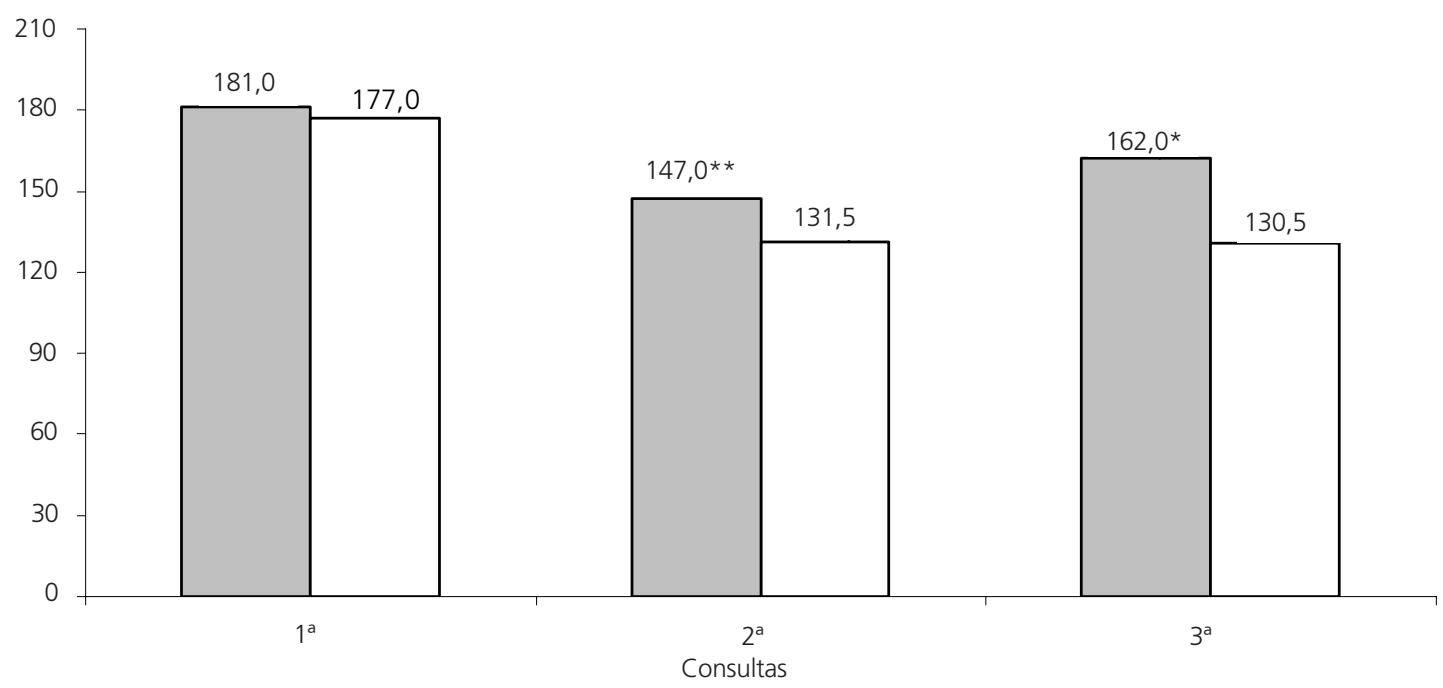

$\square$ Obesos $\quad \square$ Não Obesos

Figura 1. Relação entre obesidade e os níveis medianos de glicemia, em 3 momentos distintos. Nota: ${ }^{*} p<0,01 ;{ }^{*} p<0,05$. 
pacientes ainda não haviam recebido orientação dietética, a obesidade parece não ter influenciado a glicemia; porém, nos atendimentos seguintes, os valores significativamente mais baixos de glicemia entre os não-obesos, sugerem que a orientação nutricional determinou um resultado melhor para estes, do que para os obesos. Este fato confirma a importância do controle de peso para a melhora do estado metabólico do diabético.

Relacionando-se a glicemia de jejum ao tempo de manifestação/duração da doença, verificou-se que, no momento inicial, não houve diferença significante da glicemia entre os indivíduos com duração da doença menor ou igual a 5 anos e os com duração maior. Porém, no segundo e terceiro momentos, as pessoas com menor período de duração do diabetes apresentaram valores significantemente menores de glicemia (Figura 2).

Quando se analisaram os 112 pacientes que apresentaram dados sobre atividade física na primeira e segunda consulta, verificou-se que esta atividade aumentou significantemente de 15,2\% para 39,3\% ( $\left.\chi^{2}=16,42 ; p<0,0001\right)$; porém, em ambas as consultas, não foram encontradas diferenças estatisticamente significantes dos níveis glicêmicos, IMC e lípides séricos, entre os que realizavam atividade física e os que não a praticavam.

O efeito da atividade física não pôde ser observado, provavelmente devido ao fato de o estudo não ter sido controlado, havendo variáveis de confusão, tais como a introdução simultânea de dieta e de medicação. No entanto, o aumento da freqüência dos praticantes de exercícios físicos pode sugerir uma maior conscientização destes diabéticos para a mudança do seu estilo de vida.

Silva \& Lima ${ }^{22}$, avaliando o efeito do exercício físico em 33 indivíduos diabéticos tipo 2 , concluíram que um programa de exercício físico, com atividades aeróbias e de resistência muscular localizada, quatro vezes por semana,

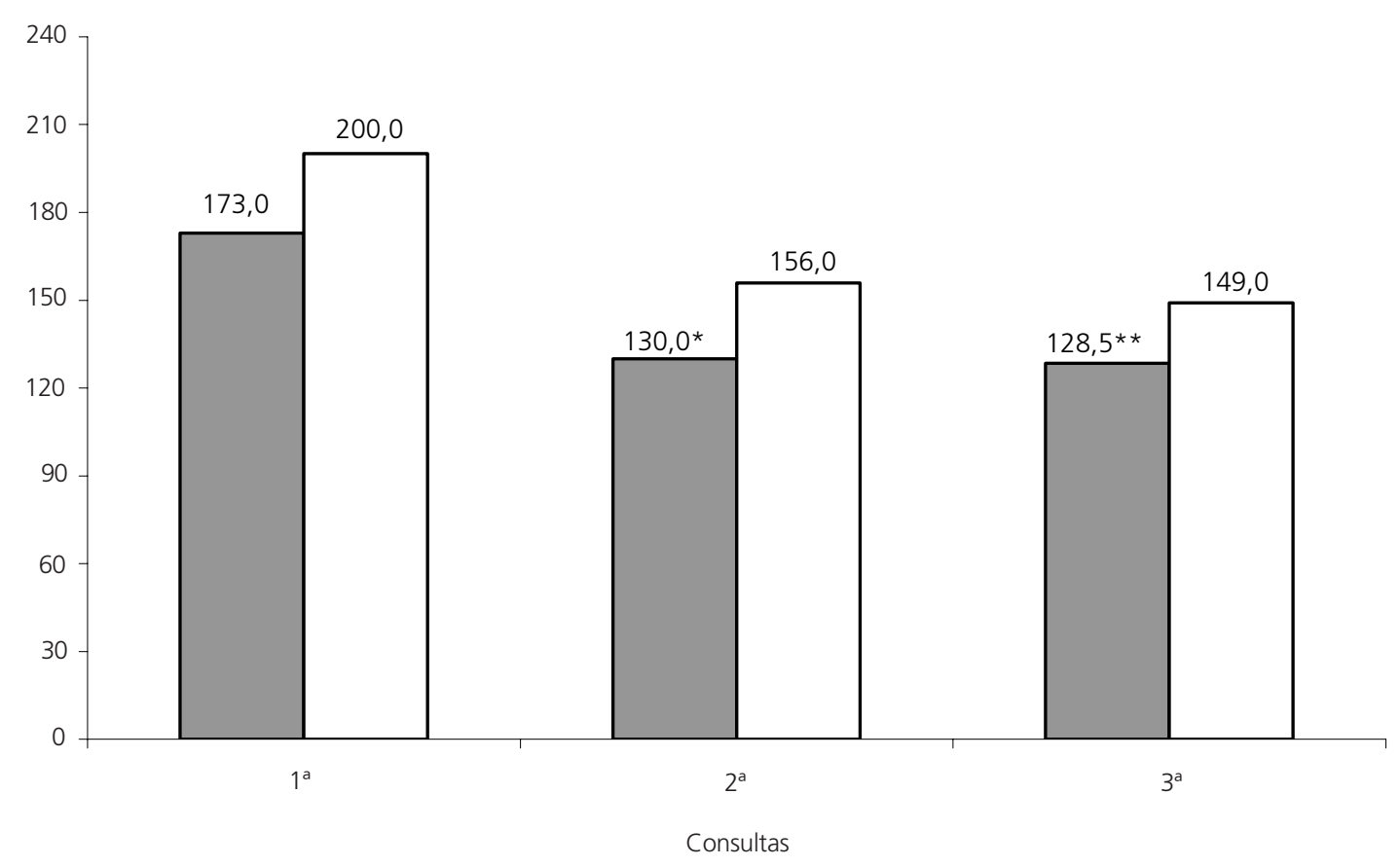

$\square$ Até 5 anos $\square>5$ anos

Figura 2. Relação entre os níveis medianos de glicemia e o tempo de duração do diabetes em 3 momentos distintos. Nota: ${ }^{*} p<0,01 ; * * p<0,05$. 
com sessões de 60 minutos, resultou em redução da glicemia de jejum, HbA1 (hemoglobina glicada), triglicerídeos e IMC, aumento do HDL-C e melhora da eficiência cardíaca, mostrando assim a importância da atividade física bem orientada.

Comparando-se os valores medianos obtidos no momento inicial e três meses após intervenção dietética, observou-se que houve redução significante de IMC, glicemia de jejum, LDL-c e triglicerídeos $(p<0,01)$ e também de colesterol total $(p<0,05)$. Não houve modificação significante do nível mediano de HDL-c (Figura 3).

Entre os 166 pacientes que tiveram exame de glicemia de jejum nos três atendimentos (inicial, 3 e 6 meses após), verificou-se que, no início, apenas 8,4\% apresentavam glicemia aceitável $(<126 \mathrm{mg} / \mathrm{dL})$; no segundo momento, este percentual subiu para 34,9\%; no terceiro, subiu para $40,4 \%$.

A redução significante dos níveis de glicemia, dos lípides séricos (colesterol total, LDL-c e triglicerídeos) e do IMC, pode vir a contribuir para a redução do risco de complicações crônicas e dos custos sociais e econômicos. Estes são, provavelmente, efeitos benéficos decorrentes da atenção multidisciplinar voltada para o controle do diabetes. São resultados consistentes com os de outros estudos que, utilizando diferentes metodologias, encontraram também resultados favoráveis ${ }^{14,23,24}$.

Leite et al. ${ }^{23}$, avaliando 78 pacientes com diabetes (tipo 1 e 2), antes e após 1 ano de acompanhamento multiprofissional, encontraram redução média da glicemia pós-prandial, de $220,1 \pm 84,0 \mathrm{mg} / \mathrm{dL}$ para $171,7 \pm 55,2 \mathrm{mg} / \mathrm{dL}$ $(p<0,01)$, além de redução da hemoglobina glicada, de $8,1 \pm 2,25 \%$ para $7,5 \pm 2,0 \%$ $(p<0,05)$. Para glicemia de jejum, colesterol total, HDL-C, LDL-c e triglicerídeos, não encontraram modificação estatisticamente significante.

Gagliardino \& Etchegoyen ${ }^{14}$ avaliaram um programa de educação em diabetes, implantado e testado na Argentina, Bolívia, Brasil, Chile, Colômbia, Costa Rica, Cuba, México, Paraguai e Uruguai. Neste programa, avaliaram 446 diabéticos tipo 2; ao final de 12 meses, observaram

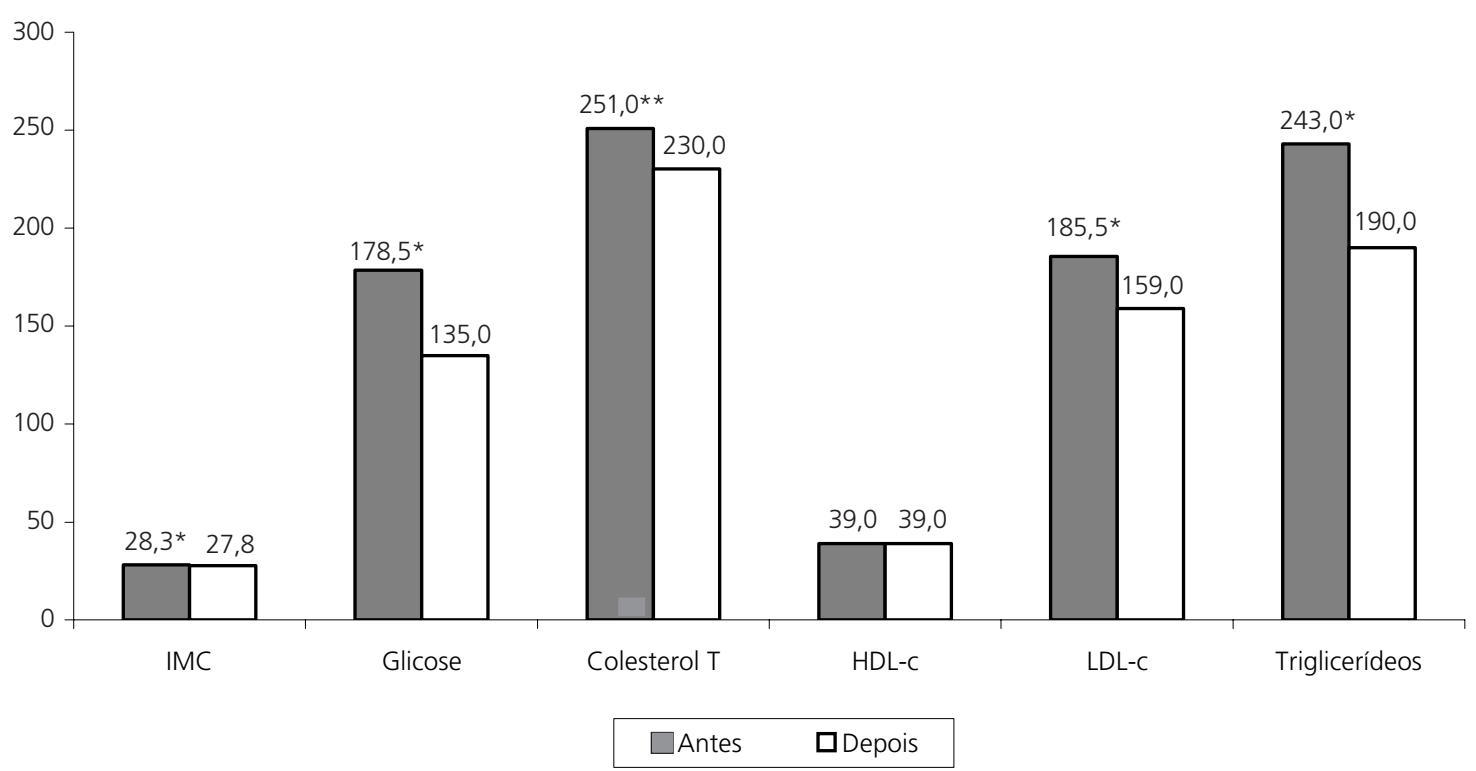

Figura 3. Comparação dos valores medianos de IMC, glicemia e lipídeos séricos, antes e após 3 meses de intervenção. Nota: ${ }^{*} p<0,01 ;{ }^{*} p<0,05$. 
redução significante $(p<0,01)$ da glicemia de jejum $(10,2 \pm 3,3 \mathrm{mmol} / \mathrm{L}$ para $8,7 \pm 3,0 \mathrm{mmol} / \mathrm{L}), \mathrm{da}$ $\operatorname{HbA} 1 \mathrm{c}(8,9 \pm 2,1 \%$ para $7,8 \pm 1,6 \%)$, do colesterol total $(6,2 \pm 0,8$ para $5,4 \pm 1,0 \mathrm{mmol} / \mathrm{L})$ e dos triglicérides $(2,8 \pm 1,7$ para $2,1 \pm 1,2 \mathrm{mmol} / \mathrm{L})$. Também observaram redução significante do peso $(83,2+14,9$ para $81,2+15,2 \mathrm{~kg})(p<0,05)$.

Araúz et al. ${ }^{24}$, em estudo realizado em El Guaco, Costa Rica, verificaram que a intervenção educativa comunitária, com a participação de pacientes diabéticos tipo 2 , seus familiares e pessoal de saúde, foi capaz de reduzir a glicemia, de $189 \pm 79 \mathrm{mg} / \mathrm{dL}$ para $157 \pm 78 \mathrm{mg} / \mathrm{dL}$ $(p=0,03)$; a hemoglobina glicada, de 11,3 $\pm 2,4 \%$ para $9,7 \pm 2,3 \%(p=0,05)$ e os triglicerídeos, de $214 \pm 108 \mathrm{mg} / \mathrm{dL}$ para $187 \pm 112 \mathrm{mg} / \mathrm{dL}$ $(p=0,04)$; porém, não encontraram mudanças significantes no peso e nos níveis de colesterol total, LDL-c e HDL-C.

Os resultados do presente estudo mostram que a atenção primária multiprofissional surtiu efeitos, contribuindo tanto para a redução do peso e a melhoria do controle glicêmico e dos lípides séricos, como para a melhoria da qualidade de vida dos pacientes. Sugerem ainda a necessidade de que esta intervenção seja feita o mais precocemente possível.

\section{REFERÊ NCIAS}

1. Reis AF, Velho G. Bases genéticas do diabetes mellitus tipo 2. Arq Bras Endocrinol Metabol. 2002; 46:426-32.

2. DeFronzo RA. Pathogenesis of type 2 diabetes: Metabolic and molecular implications for identifying diabetes genes. Diabetes Rev. 1997; 5:177-269.

3. Araújo RB, Santos I, Cavaleti MA, Costa JSD, Béria JU. Avaliação do cuidado prestado a pacientes diabéticos em nível primário. Rev Saúde Pública. 1999; 33:24-32.

4. Malerbi DA, Franco LJ. Multicenter study of the prevalence of diabetes mellitus and impared glucose tolerance in the urban Brazilian population aged 30-69 years. Diabetes Care. 1992; 15(11): 1509-16.

5. Brasil. Ministério da Saúde. Plano de reorganização da atenção à hipertensão e ao diabetes mellitus: fase de detecção de casos suspeitos de DM.
Informes Técnicos Institucionais. Rev Saúde Pública. 2001; 35(5):490-3.

6. Gross JL, Nehme M. Detecção e tratamento das complicações crônicas do diabetes melito: Consenso da Sociedade Brasileira de Diabetes e Conselho Brasileiro de Oftalmologia. Rev Ass Med Bras; 1999; 45(3):279-84.

7. United Kingdom Prospective Diabetes Study Group. Intensive blood-glucose control with sulfonylureas or insulin compared with conventional treatment and risk of complications in patients with diabetes type 2; UKPDS 33. Lancet. 1998; 352(9131):837-53.

8. World Health Organization. Study Group on Diabetes mellitus. Technical Report Series, 727. Second report. Geneva; 1985.

9. World Health Organization. Preventing and managing the global epidemic. Report of a Who Consultation on Obesity. Geneva; 1998; 3:6-15.

10. Consenso Brasileiro sobre Diabetes. Diagnóstico e classificação do diabetes mellitus e tratamento do diabetes mellitus tipo 2. São Paulo: Sociedade Brasileira de Diabetes; 2000.

11. Centers for Disease Control \& Prevention. Epi-info, version 6: A Word processing, database, and statistics program for Public Health. Geneva: World Health Organization, 1997.

12. Fox E, Kuo J, Tilling L, Ulrich C. User's manual Sigma Stat: Statistical software for Windows. Jandel; 1994.

13. Valle T, Koivisto VA, Reunanen A, Kangas T, Rissanen A. Glycemic control in patients with diabetes in Finland. Diabetes Care. 1999; 22(4):575-9.

14. Gagliardino JJ, Etchegoyen G. A model educational program for people with type 2 diabetes: a cooperative Latin American implementation study (PEDNID-LA). Diabetes Care. 2001; 24(6):1001-7.

15. Schumacher MC, Smith KR. Diabetes in Utah among adults: Interaction between diabetes and other risk factors for microvascular and macrovascular complications. Am J Public Health. 1988; 78(9):195-201.

16. Assunção MCF, Santos IS, Gigante DP. Atenção primária em diabetes no sul do Brasil: estrutura, processo e resultado. Rev Saúde Pública. 2001; 35(1):88-95.

17. Goldenberg P, Franco LJ, Pagliaro H, Silva RS, Santos CA. Diabetes mellitus auto-referido no município de São Paulo: prevalência e desigualdade. Cad Saúde Pública 1996; 12(1):37-45.

18. Fausto Neto $A M Q$. Família operária e reprodução da força de trabalho. Petrópolis: Vozes; 1982.

19. Dorman JS, Tajima N, La Porte RE, Becher DJ, Cruickshanks KJ, Wagener DK, et al. The Pittsburgh 
insulin-dependent diabetes mellitus (IDDM) morbidit and mortality study: case-control analyses of risk factors for mortality. Diabetes Care. 1985; 8 Suppl 1:S54-60.

20. Batista MCR, Franceschini SCC, Priore SE, Avaliação de indicadores antropométricos de adultos e idosos brasileiros. Nutrire: Rev Soc Bras Alim Nutr. 2002; 23:67-78.

21. Cabrera MAS, Jacob Filho W. Obesidade em idosos: prevalência, distribuição e associação com hábitos e co-morbidades. Arq Bras Endocrinol Metab. 2001; 45(5):494-501.

22. Silva CA, Lima WC. Efeito benéfico do exercício físico no controle metabólico do diabetes mellitus tipo 2 a curto prazo. Arq Bras Endocrinol Metabol. 2002; 46(5):550-6.
23. Leite SAO, Costa PAB, Guse C, Dorociaki JC, Silveira MC, Teodorovicz R, Martinatto JS, Niclewicz EA. Enfoque multidisciplinar ao paciente diabético: avaliação do impacto do "Staged Diabetes Management" em um sistema de saúde privado. Arq Bras Endocrinol Metabol. 2001; 45(5): 481-6.

24. Araúz AG, Sanchez G, Padilla G, Fernández $M$, Rosello M, Gusmán S. Intervención educativa comunitaria sobre el diabetes en el ámbito de la atención primaria. Rev Panam Salud Publica. 2001; 9(3):145-53.

Recebido para publicação em 8 de março e aceito em 26 de maio de 2004. 